\title{
Domain movements of NADPH-cytochrome P450 oxidoreductase (CPR) are required for the smooth electron transfer from CPR to heme-heme oxygenase-1 (HO-1) complex
}

\author{
M. Sugishima ${ }^{1}$, J. Taira ${ }^{2}$, M. lijima ${ }^{3}$, H. Sato ${ }^{1}$, K. Wada ${ }^{4}$, M. Noguchi' ${ }^{1}$ K. Fukuyama ${ }^{5}$, M. Takano ${ }^{3}$, H. Sakamoto ${ }^{2}$, \\ K. Yamamoto ${ }^{1}$
}

\author{
${ }^{1}$ Dept. of Med. Biochem., Kurume Univ. Sch. Med., 67 Asahi-machi, Kurume 830-0011, Japan, \\ ${ }^{2}$ Dept. of Biosci. and Bioinfo., Grad. Sch. of Comp. Sci. and Sys. Engineer., Kyushu Inst. Tech., \\ 680-4 Kawazu, Iizuka 820-8502, Japan, \\ ${ }^{3}$ Dept. of Pure and Applied Physics, Waseda Univ., Tokyo 169-8555, Japan, \\ ${ }^{4}$ Dept. of Med. Sci., Univ. of Miyazaki, 5200 Kihara, Miyazaki 889-1601, Japan, \\ ${ }^{5}$ Dept. Biol. Sci., Grad. Sch. of Sci., Osaka Univ., 1-1 Machikaneyama-cho, Toyonaka 560-0043, Japan. \\ sugishima_masakazu@med.kurume-u.ac.jp
}

Heme oxygenase-1 (HO-1) catalyzes the heme degradation using seven electrons supplied by NADPH-cytochrome P450 oxidoreductase (CPR) where FAD and FMN are bound as co-enzymes. Electrons flow from NADPH to heme in the redox partner via FAD and FMN. Previous biophysical analyzes such as SAXS and FRET suggest the existence of a dynamic equilibrium between the open and the closed forms of CPR in which orientations of FMN and FAD-binding domains are different [1]. We previously determined the crystal structure of the open-form stabilized CPR ( $\triangle \mathrm{TGEE}$ ) in complex with heme-HO-1 at $4.3 \AA$ resolution and demonstrated that $\triangle$ TGEE is tightly bound to heme-HO-1 while the reduction in heme-HO-1 using $\triangle$ TGEE is markedly slow because FAD is too far from FMN for electron transfer between them [2].

Here we characterized the enzymatic activity and the reduction kinetics of HO-1 using the closed-form stabilized CPR (147CC514) where the disulfide bond between FAD and FMN binding domains was introduced. We also analyzed the interaction between 147CC514 and heme-HO-1 by analytical ultracentrifugation [3]. The results indicate that HO-1 activity coupled with $147 \mathrm{CC} 514$ is markedly weaker than that coupled with CPR and the interaction between 147CC514 and heme-HO-1 is considerably weak. In addition, we examined the coupling of the redox and the structural states by full-scale molecular dynamics (MD) simulation of CPR (total $86.4 \mu \mathrm{s}$ ) [4]. Our MD result demonstrated that CPR has a tendency to open in the fully-reduced state while the major form of CPR is the closed form both in the fully-oxidized and fully-reduced states. We also found a correlation between the FAD-FMN distance and the predicted FMN-HO-1 distance, which is embedded in the equilibrium thermal fluctuation of CPR. Thus, the redox coupled transition between the open and the closed forms of CPR is indispensable for the smooth electron transfer from CPR to hemeHO-1 complex.

Further, we prepared the fusion protein of $\triangle \mathrm{TGEE}$ and HO-1 referring to the previously reported structure of $\triangle \mathrm{TGEE}$ in complex with heme-HO-1 and determined its fusion protein structure in complex with heme at $3.25 \AA$ resolution [5]. Unexpectedly, no NADP ${ }^{+}$was $^{2}$ observed in the fusion protein structure although $\mathrm{NADP}^{+}$was contained in the crystallization droplets and $\mathrm{NADP}^{+}$was observed in the previous complex structure of $\triangle \mathrm{TGEE}$ and heme-HO-1. Because the structural features of the $\mathrm{NADP}^{+}$-free form of $\mathrm{CPR}$ were also observed in the fusion protein structure, the fusion protein structure reflects the $\mathrm{NADP}^{+}$-free form of $\triangle \mathrm{TGEE}-\mathrm{heme}-\mathrm{HO}-1$ complex. Structural comparison of the $\mathrm{NADP}^{+}$-bound $\triangle \mathrm{TGEE}-$ heme-HO-1 complex and the $\mathrm{NADP}^{+}$-free fusion protein suggests that $\mathrm{NADP}^{+} / \mathrm{NADPH}$ binding regulates the conformation change of the FAD-binding domain of CPR, which may control the efficiency of the electron transfer from FMN to heme-HO-1.

[1] Iyanagi, T., Xia, C. \& Kim, J. J. P. (2012) Arch. Biochem. Biophys. 528, 72.

[2] Sugishima, M., Sato, H., Higashimoto, Y., Harada, J., Wada, K., Fukuyama, K. \& Noguchi, M. (2014) Proc. Natl. Acad. Sci. USA 111, 2524.

[3] Sugishima, M., Taira, J., Sagara, T., Nakao, R., Sato, H., Noguchi, M., Fukuyama, K., Yamamoto, K., Yasunaga, T. \& Sakamoto, H. (2020) Antioxidants 9, 673 .

[4] Iijima, M., Ohnuki, J., Sato, T., Sugishima, M. \& Takano, M. (2019) Sci. Rep. 9, 9341.

[5] Sugishima, M., Sato, H., Wada, K. \& Yamamoto, K. (2019) FEBS Lett. 593, 868.

\section{Keywords: Heme metabolism; Electron transfer; Domain motion; Cofactor binding}

We acknowledged Mr. Sagara and Ms. Takemoto of Kyushu Inst. Tech., and Dr. Ohnuki and Dr. Sato of Waseda Univ. for analytical centrifugation and MD experiments, respectively. We also acknowledged beamline staffs of BL44XU, SPring-8 for crystallographic data collection. This work was partially supported by Kakenhi Grant numbers 25840026, 16K07280, and 19K06515 from JSPS, and grants from Takeda Science Foundation and Protein Research Foundation.

Acta Cryst. (2021), A77, C1130 